\title{
Cationic functionalisation of cellulose using a choline based ionic liquid analogue $\dagger$
}

\author{
Andrew P. Abbott, ${ }^{* a}$ Thomas J. Bell, ${ }^{a}$ Sandeep Handa ${ }^{a}$ and Barry Stoddart ${ }^{b}$ \\ Received 11th April 2006, Accepted 26th June 2006 \\ First published as an Advance Article on the web 4th July 2006 \\ DOI: $10.1039 / \mathrm{b605258d}$
}

The efficient cationic functionalisation of cellulose is demonstrated using an ionic liquid analogue, based on a eutectic mixture of a choline chloride derivative and urea, which acts as both solvent and reagent. It was determined that all the available hydroxyl groups on cellulose had been modified.

\section{Introduction}

The cationic functionalisation of cellulose is of considerable industrial importance and has found applications in the paper industry, cosmetics, textiles, in flotation and flocculation and in drilling fluids. ${ }^{1}$ In particular, it is used in the removal of acidic dyes from aqueous effluent produced by the textile industry, which significantly reduces the environmental impact of such processes. ${ }^{2}$ These dyes are of an anionic nature comprising of groups such as sulfonates, carboxylates or sulfates, and so suit the use of a cationic substrate. ${ }^{3}$ The form of cellulose often used, cotton, is natural, inexpensive and renewable. ${ }^{3}$

The preferred route to the formation of these materials is by the etherification of cellulose using glycidyl ammonium salts or alkylene epoxides in the presence of a suitable alkaline catalyst, usually sodium hydroxide. Cationic substitution values between 0.034 and 0.5 moles of quaternary nitrogen per mole of glucose unit have been reported. ${ }^{4-6}$ However, such approaches entail the use of large amounts of organic solvents, and result in low yields and the loss of product as a result of solubility in the reaction mixture. ${ }^{1}$ Two other approaches, coupling oligo-ionomers to cellulose fibres and grafting on cationic polymer chains, have also been reported, but again yields tend to be poor. ${ }^{7}$

We have previously reported the development of ionic liquids based on a combination of the inexpensive and readily available components choline chloride $\left(\mathrm{ChCl} ; \mathrm{HOCH}_{2} \mathrm{CH}_{2} \mathrm{~N}(\mathrm{Me})_{3} \mathrm{Cl}\right)$ and zinc chloride as alternatives to the more commonly employed alkyl imidazolium-aluminium chloride mixtures. ${ }^{8}$ These new Lewis acidic solvents have been successfully employed for a variety of reactions. ${ }^{9-11} \mathrm{We}$ have also reported a eutectic mixture of choline chloride and urea which forms an ionic liquid analogue with the advantage of being non-toxic and readily biodegradable but has no Lewis acidity. ${ }^{12}$ Two uses of this mixture for inorganic applications have recently been reported. ${ }^{13,14}$ In this current study

${ }^{a}$ Department of Chemistry, University of Leicester, Leicester, UK

LE17RH.E-mail: apa1@le.ac.uk; Fax:+44 (0)116 252 3789;

Tel: +44 (0)116252 2087

${ }^{b} P \& G$ Technical Centres, Whitley Road, Longbenton, Newcastle Upon

Tyne, UK NE12 9TS

$\uparrow$ Electronic supplementary information (ESI) available: Experimental

procedures and I.R. calibration plot. See DOI: 10.1039/b605258d we report the first successful use for organic synthetic processes of a deep eutectic solvent, consisting of a choline derivative and urea, which can be employed as both a reagent and solvent for the cationic functionalisation of cellulose.

\section{Results and discussion}

Cellulose, in the form of cotton wool, was quaternised using sodium hydroxide and a chlorcholine chloride-based $(\mathrm{ClChCl}$; $\left.\mathrm{ClCH}_{2} \mathrm{CH}_{2} \mathrm{~N}(\mathrm{Me})_{3} \mathrm{Cl}\right)$ deep eutectic solvent, for $15 \mathrm{~h}$ at $90{ }^{\circ} \mathrm{C}$ (Scheme 1). $\uparrow$ The modified cotton wool was removed from the solvent and copiously washed with water to give the product. A variety of methods for determining the degree of cationic functionalisation of cellulose substituted by conventional means have been reported. Kjeldahl analysis accurately determines the nitrogen content and thus the degree of cationic substitution, ${ }^{1}$ although other approaches such as solid-state NMR,,${ }^{15}$ elemental analysis $^{16}$ and dye adsorption ${ }^{17}$ have been reported. Analysis of the product by the Kjeldahl method gave a cationic substitution value of $0.5 \%$ of quaternary nitrogen per mole of glucose unit. Cationic functionalisation was also confirmed by the use of a cationic dye, methylene blue. A calibration plot was constructed by using different concentrations of methylene blue and this was used to measure the dye concentration in the supernatant liquid resulting from placing $0.05 \mathrm{~g}$ of substituted cotton wool in $3 \mathrm{ml}$ of dye solution for 3 hours (see ESI $\dagger$ ). This was repeated with unmodified cotton wool and the concentration of dye repelled due to the functionalisation was determined to be $1.44 \times 10^{-8}$ moles.

The above analysis assumes that all the quaternised groups become attached to the cellulose by reaction of the chlorcholine chloride with the available $\mathrm{OH}$ groups, and that no physisorption onto the cotton wool is occurring. The extent to which physisorption could be occurring was examined by repeating the reaction without the use of sodium hydroxide, thus preventing the chemical reaction from occurring. Dye analysis of the product showed that $8.25 \times 10^{-9}$ moles of dye were repelled, thus confirming the presence of functionalised material. By reference to

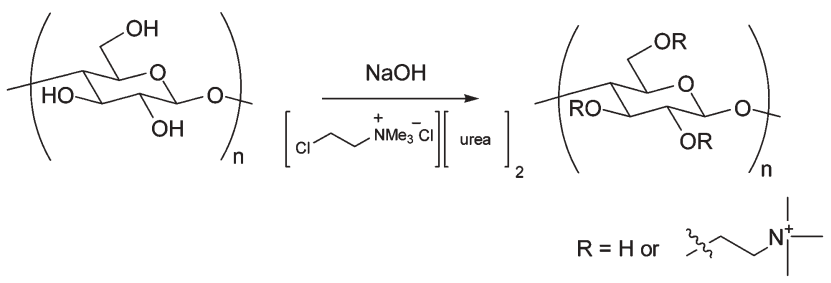

Scheme 1 Derivatisation of cellulose with cationic functionalities. 
the Kjeldahl analysis and assuming that the repulsion of the cationic dye follows a linear relationship, this equates to $0.28 \%$ of quaternary nitrogen per mole of glucose unit.

Samples of the physisorbed material and chemi/physisorbed material were washed with water for varying periods of time (0 to 72 hours) to determine the extent of leaching of quaternised material (Fig. 1). This showed that cellulose containing only physisorbed material progressively loses all its quaternised material whereas the cellulose containing both chemi- and physisorbed material loses around $50 \%$.

Chemisorbed material therefore accounts for around half of the quaternised product. As the cotton wool was washed thoroughly in the work-up procedure the material removed over the period of 72 hours can be assumed to be genuinely physisorbed rather than that merely trapped between the cotton wool fibres.

Secondary Ion Mass Spectrometry (SIMS) showed peaks relating to both chemisorbed and physisorbed material. Taking into account the extent of physisorption, the degree of cationic substitution based only on chemisorbed material was estimated to be $0.22 \%$,

The degree of cationic substitution reported is based on the assumption that all of the hydroxyl groups on the cellulose fibres are available for functionalisation to take place. However, only the hydroxyl groups on the surface of the fibres are likely to be accessible for substitution. The ratio of the surface groups to those in the bulk of the monomer was estimated using the following equation. $\dagger$

$$
\text { Ratio }=\frac{\pi(r+2 R)^{2}-\pi r^{2}}{\pi(r+2 R)^{2}}
$$

Where $r$ is the radius of the cellulose fibre and $R$ is the radius of the glucose monomer on the surface of the fibre. The radius of the cellulose fibre $\left(1 \times 10^{-5} \mathrm{~m}\right)$ was determined by the use of atomic force microscopy, and the radius of a glucose monomer $(7.95 \times$ $10^{-10} \mathrm{~m}$ ) by a molecular modelling package. ${ }^{18}$ The resulting ratio was found to be $3.18 \times 10^{-4}$, or $0.0318 \%$ of available glucose monomers and hence the available hydroxyl groups present. This figure lies just outside the range of the cationic substitution determined by Kjeldahl analysis. However, the estimation of the

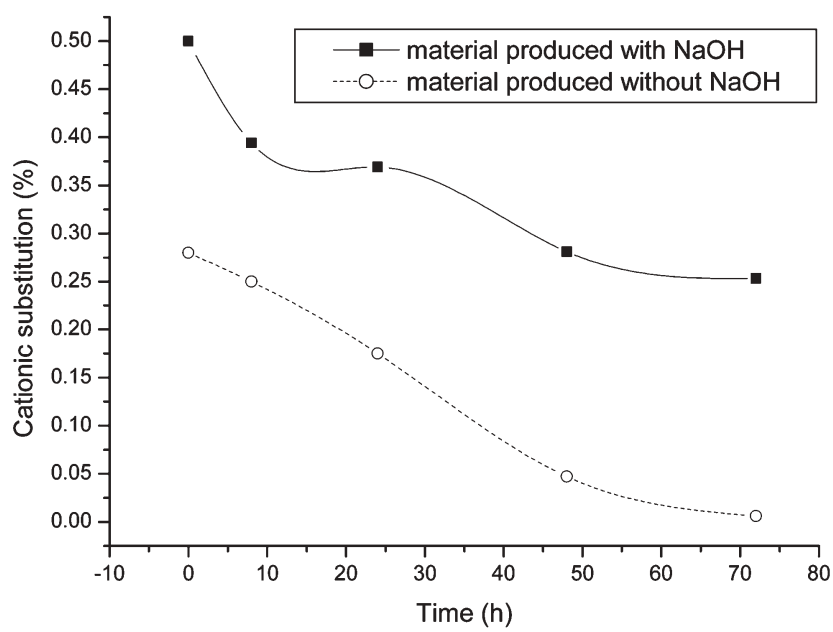

Fig. 1 Comparison of derivatised cellulose produced with and without the use of $\mathrm{NaOH}$.

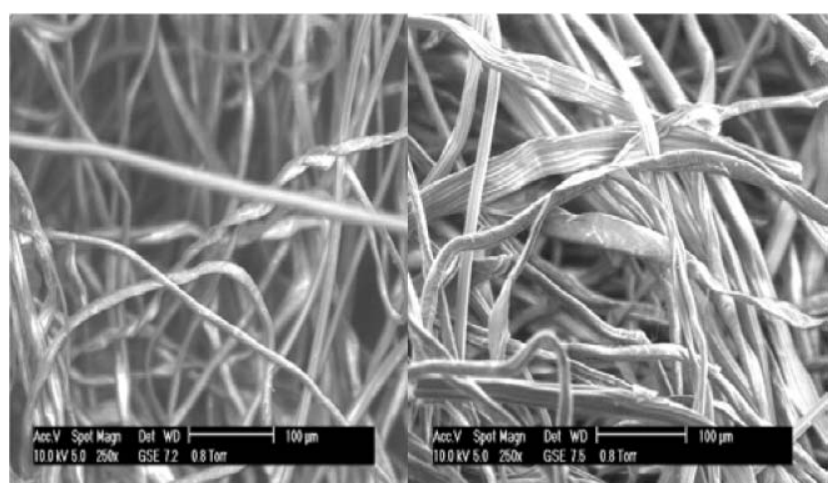

Fig. 2 SEM images of cotton wool (left) and cotton wool after contact with eutectic solvent for 15 hours at $90{ }^{\circ} \mathrm{C}$.

available groups on the surface was based on the assumption that no wetting (opening up) of the cellulose fibres takes place.

Fig. 2 shows scanning electron microscopy (SEM) images of cotton wool before and after being subjected to the deep eutectic solvent. These show that the cotton wool is slightly wetted upon contact with the eutectic solvent presumably resulting in more hydroxyl groups being available for reaction. Taking this into account it is reasonable to assume that the vast majority, if not all, of the available hydroxyl groups on the cellulose have been functionalised.

The cationic functionalisation of the surface leads to a material with increased hydrophilicity. Thermogravimmetry showed that the sample was able to absorb an additional $26 \mathrm{wt} \%$ of water compared to unmodified cellulose.

The effect of different reaction times (Table 1, entries 1-3) and temperatures (entries 2, 4 and 5) were investigated to discover the optimum conditions for the reaction. The cationic substitution values were calculated using the dye method and are based only on chemisorbed material assuming, as previously, that around $50 \%$ of the material was due to physisorption.

From this it was concluded that 15 hours at $90{ }^{\circ} \mathrm{C}$ were the most favourable conditions for the cationic functionalisation of cellulose in the deep eutectic solvent, $\mathrm{ClChCl}$ (urea) $)_{2}$.

While cellulose derivatised with cationic functionalities repels cationic dyes it is very effective at adsorbing anionic dyes, which are the main branch of water soluble dyes used in printing. The efficacy of using cationic functionalised cellulose for dye adsorption was determined using orange II. It was found that the modified cellulose produced in entry 2 of Table 1 was capable of extracting $0.06 \mathrm{wt}^{\%} \%$ orange II dye, which suggests that these materials may be suitable alternatives for cleaning dyes from aqueous waste streams.

Table 1 Determination of the optimum conditions for the cationic functionalisation of cellulose in a deep eutectic solvent

\begin{tabular}{lccl}
\hline Entry & Time/h & Temperature $/{ }^{\circ} \mathrm{C}$ & Cationic substitution $(\%)$ \\
\hline 1 & 3 & 90 & 0.09 \\
2 & 15 & 90 & 0.22 \\
3 & 60 & 90 & 0.19 \\
4 & 15 & 60 & 0.06 \\
5 & 15 & 120 & 0.19 \\
\hline
\end{tabular}




\section{Conclusion}

This work has shown that an ionic liquid analogue based on $\mathrm{ClChCl}$ and urea acts as both reagent and solvent in the effective cationic functionalisation of cellulose. It was determined that all the avaliable hydroxyl groups on cellulose had been modified and that this material is significantly more hydrophilic than cellulose. We have demonstrated the potential to produce a material with a green application from renewable sources using a green synthetic methodology.

\section{Acknowledgements}

The authors would like to acknowledge EPSRC, Crystal Faraday and Procter and Gamble for funding this work.

\section{Notes and references}

1 R. S. Hasselroth, D. B. Marktbreit and M. H. Eschborn, U.S. Pat. 4,940,785, 1990.

2 J. A. Laszlo, Am. Dyest. Rep., 1994, 17.
3 I. Bouzaida and M. B. Rammah, Mater. Sci. Eng., 2002, 21, 151.

4 F. W. Stone and J. M. Rutherford, U.S. Pat 3,472,840, 1969.

5 R. L. Kreeger and S. Zhou, Int. Pat. WO 2005/000903 Al, 2005.

6 P. M. Van der Horst, Int. Pat. WO2005/061792 A1, 2005.

7 E. Gruber, C. Ganzow and T. Ott, ACS Symp. Ser., 1998.

8 A. P. Abbott, G. Capper, D. L. Davies and R. K. Rasheed, Inorg. Chem., 2004, 43, 3447.

9 A. P. Abbott, G. Capper, D. L. Davies, R. Rasheed and V. Tambyrajah, Green Chem., 2002, 4, 24.

10 R. C. Morales, V. Tambyrajah, P. R. Jenkins, D. L. Davies and A. P. Abbott, Chem. Commun., 2004, 158.

11 A. P. Abbott, T. J. Bell, S. Handa and B. Stoddart, Green Chem., 2005, 7, 705 .

12 A. P. Abbott, G. Capper, D. L. Davies, R. K. Rasheed and V. Tambyrajah, Chem. Commun., 2003, 70.

13 J.-H. Liao, P.-C. Wu and Y.-H. Bai, Inorg. Chem. Commun., 2005, 8, 4 , 390.

14 E. R. Cooper, C. D. Andrews, P. S. Wheatley, P. B. Webb, P. Wormald and R. E. Morris, Nature, 2004, 430, 7003, 1012.

15 P. Aggarwal and D. Dollimore, Thermochim. Acta, 1997, 291, 65.

16 G. S. Chouhan, B. Singh and S. K. Dhiman, J. Appl. Polym. Sci., 2004, 91, 2454.

17 A. Hashem and R. M. El-Shishtawy, Adsorpt. Sci. and Technol., 2001, 19, 3, 197.

18 Spartan Pro, Wavefunction Inc., Irvine, CA, USA. 